\section{Resultados de um programa de triagem auditiva neonatal em Maceió}

\section{Results of a neonatal hearing screening program in Maceió} Keywords: hearing, hearing loss, prevention.

Palavras-chave: audição, deficiência auditiva, prevenção Antônio Lira dos Anjos', Elizângela Dias Camboim $^{3}$, Marcella de Carvalbo Ramos Pimentel ${ }^{4}$

Resumo / Summary

$\mathrm{D}$ esde 1998, com a criação do grupo de apoio a triagem auditiva neonatal, vários programas de triagem auditiva foram implantados no país. Em Alagoas, o primeiro programa foi criado em 2003, do qual não se publicou nenhum resultado. Sabe-se que a audição é importante para a comunicação humana, pois a perda auditiva na criança pode acarretar distúrbios na aquisição da fala, na linguagem e no desenvolvimento emocional, educacional e social. Objetivo: Apresentar os resultados obtidos em um programa de triagem auditiva neonatal em Maceió. Material e Método: Trata-se de um estudo analítico retrospectivo para analisar exames realizados entre setembro de 2003 e dezembro de 2006 em um hospital privado de Maceió. Resultados: De 2002 recém-nascidos, 1626 atenderam aos critérios de inclusão, sendo 835 (51,4\%) do sexo masculino. A triagem auditiva foi adequada em 1416 casos $(87,1 \%)$, sendo a faixa etária mais freqüente entre 16 e 30 dias. No total, 163 (10,0\%) apresentavam indicadores de risco para deficiência auditiva, o mais freqüente foi a hiperbilirrubinemia. Conclusões: Os resultados estatísticos obtidos neste programa de triagem auditiva demonstram a importância da implantação e manutenção de um programa dessa natureza. Esta análise foi considerada importante para contribuição de um estudo multinacional ou regional.

\begin{abstract}
Since 1998, after we started the support group for neonatal hearing screening, many other hearing screening programs were held in Brazil. In Alagoas, the first program started in 2003, but none of its results were published. Hearing is paramount for human communication; therefore, childhood hearing loss can impair speech acquisition, emotional, educational and social development. Aim: to present the results achieved in a neonatal hearing screening program in Maceió. Materials and Methods: a retrospective analytical study was carried out in order to study the results from tests carried out from September 2003 to December 2006 in a private hospital of Maceió. Results: from a total of 2002 newborns, 1,626 fitted the inclusion criteria, 835 (51.4\%) males. The hearing screening was considered appropriate in 1416 cases (87.1\%), and the most frequently found age was between 16 and 30 days. Finally, 163 (10.0\%) children presented risk indicators for hearing loss, and hyperbilirubinemia was the most common indicator. Conclusions: statistical results obtained from this hearing screening program show the importance of holding such programs. This study is important because it contributes to further regional or multinational studies.
\end{abstract}




\section{INTRODUÇÃO}

A habilidade de comunicação é um traço distintivo da existência humana, sendo um dos maiores contribuintes para o bem estar de qualquer indivíduo. Dentro desse contexto, a audição desempenha um papel fundamental, uma vez que é considerada a pedra angular sobre a qual se constrói o intrincado sistema da comunicação humana ${ }^{1}$. Neste sentido, a privação sensorial auditiva na criança compromete não só a sua comunicação, mas seu potencial de linguagem receptiva e expressiva, sua alfabetização (leitura e escrita), seu desempenho acadêmico, seu desenvolvimento emocional e social ${ }^{2}$.

Os primeiros dois anos de vida da criança têm sido considerados como um período crítico para a aquisição e desenvolvimento das habilidades auditivas e da linguagem. Uma das principais razões para isso é o fato de o sistema nervoso central apresentar grande plasticidade quando precocemente estimulado, principalmente até os 6 meses de idade, aumentando o número de conexões nervosas e conseqüentemente melhorando a reabilitação das vias auditivas ${ }^{3}$.

O Comitê Brasileiro sobre Perdas Auditivas na Infância $(\mathrm{CBPAI})^{4}$ recomenda que haja a implantação da Triagem Auditiva Neonatal Universal (TANU). O teste deve ser realizado em todas as crianças, ao nascer ou no máximo até os 3 meses de idade e, no caso de deficiência auditiva confirmada, devem receber intervenção até os 6 meses. O principal objetivo da triagem auditiva é identificar os casos prováveis de desordens auditivas de importância médica e/ou educacional5.

A implementação do programa de triagem auditiva é viável devido ao baixo custo e à facilidade de execução sob treinamento e supervisão ${ }^{6}$. Os resultados obtidos com estes programas são bastante relevantes, como por exemplo, o programa de prevenção e identificação precoce dos distúrbios da audição de umaUniversidade deSão Paulo. Neste estudo a população foi subdividida em dois grupos: de alto e de baixo risco auditivo. Na população de alto risco, foi possível identificar, durante o primeiro ano de vida, cerca de $2,5 \%$ de casos de perda auditiva sensório-neural, de grau leve a profundo. A idade média de diagnóstico audiológico foi 6,6 meses de vida e 9,8 meses para a intervenção (prótese e terapia). Na população de baixo risco, foi possível identificar perda auditiva sensório-neural em $0,85 \%$ da população5.

O teste para avaliação objetiva do sistema auditivo periférico pré-neural, com grande aplicabilidade clínica é o registro das Emissões Otoacústicas (EOAs). Estas são um tipo de energia acústica, descritas por $\mathrm{KEMP}^{7}$, geradas pela contratividade das células ciliadas externas durante o mecanismo ativo da função coclear que se propagam à orelha média e conduto auditivo externo onde podem ser captadas ${ }^{8}$.
As EOAs parecem ser o melhor procedimento de triagem para a detecção precoce da deficiência auditiva, pois se trata de uma técnica rápida, não-invasiva, de fácil interpretação e de alta especificidade de sensibilidade que observa as respostas da cóclea sem depender da maturidade do sistema nervoso central ${ }^{9}$. As emissões otoacústicas mais utilizadas clinicamente são as por estímulo transiente e as produtos de distorção, sendo que as transientes são as mais recomendadas para a Triagem Auditiva Neonatal (TAN) por ser de mais rápida execução e por detectar perdas auditivas maiores que $35 \mathrm{dBNA}^{10,11}$. Esta técnica representa um importante avanço na pesquisa da perda de audição em recém-nascidos normais e de risco para deficiência auditiva ${ }^{2,7,8,10,12-15}$.

Com o crescente aumento do número de Programas de Triagem Auditiva Neonatal Universal (PTANU), houve a preocupação de traçar princípios e referenciais para controlar a efetividade dos programas. O princípio 1 destaca que todo lactente deve ter acesso à triagem auditiva por medida fisiológica. Os princípios 2, 3 e 4 abordam, respectivamente, o diagnóstico (até 3 meses), a intervenção (até 6 meses) e o monitoramento auditivo. O princípio 5 trata dos direitos da criança e da família, garantidos através de escolha informada, decisão e consentimento. O princípio 6 garante que as informações da criança, da família e dos resultados dos testes sejam sigilosos. O princípio 7 determina que sistemas de informação sejam utilizados para medir e retratar os impactos nos serviços de triagem auditiva neonatal. Por fim, o princípio 8 estipula que os programas de triagem auditiva neonatal devam prover dados para monitorar a qualidade, determinar custo efetivo, mobilizar e manter suporte na comunidade, de acordo com a legislação e regulamentação ${ }^{16}$.

Desde 1998, com a criação do grupo de apoio a triagem auditiva neonatal, vários programas de triagem auditiva foram implantados no país ${ }^{11}$. Em Alagoas, o primeiro programa foi criado em 2003, porém ainda não foi notificado registros de publicações sobre o tema no estado. Assim, o presente trabalho, objetivou apresentar os resultados obtidos em um programa de triagem auditiva neonatal na cidade de Maceió-AL, uma vez que, até o presente momento não há dados estatísticos que descrevam tais resultados.

\section{MATERIAL E MÉTODO}

Foi realizado um estudo observacional analítico retrospectivo, de corte transversal, no qual foram analisados os exames realizados no período de setembro de 2003 a dezembro de 2006 contidos no banco de dados de um hospital do setor privado da cidade de Maceió-AL. Este estudo foi aprovado pelo comitê de ética da instituição sob número 666.

Foram incluídos na amostra os exames de recémnascidos com idade inferior a 3 meses e excluídos os 
exames não-analisáveis e/ou fora do critério passa/falha utilizado pelo Grupo de Apoio a Triagem Auditiva Neonatal Universal (GATANU) ${ }^{11}$, que será detalhado adiante. A coleta dos dados realizou-se por meio da análise dos exames arquivados no computador do Hospital. Além de analisar os resultados das Emissões Otoacústicas por Estímulo Transiente (EOAT), foram analisadas as correlações dos mesmos com a idade, o gênero e os Indicadores de Risco para Deficiência Auditiva (IRDA) que foram relatados e registrados.

Os indicadores de risco para deficiência auditiva levados em consideração no estudo foram: história familiar de deficiência auditiva congênita; infecção congênita (sífilis, toxoplasmose, rubéola, citomegalovírus, herpes e AIDS); consangüinidade, anomalias crânio-faciais, incluindo as alterações morfológicas de pavilhão auricular e conduto auditivo externo; peso ao nascimento inferior a $1500 \mathrm{~g}$ ou pequeno para idade gestacional (PIG); hiperbilirrubinemia; medicação ototóxica, incluindo, mas não se limitando aos aminoglicosídeos, utilizada ou não em associação aos diuréticos; meningite bacteriana; boletim Apgar de 0-4 no 1을 minuto ou 0-6 no 5을 minuto; ventilação mecânica por período maior que 5 dias; síndromes associadas à deficiência auditiva condutiva ou sensório-neural; neonatos que ficam mais que 48 horas na Unidade de Terapia Intensiva (UTI) e infecções neonatais ${ }^{16,17}$.

Os dados coletados foram de recém-nascidos submetidos à triagem auditiva neonatal por meio da captação das emissões otoacústicas por estímulo transiente, utilizando o analisador de EOA Capella, para analisar a integridade da função coclear e descartar a possibilidade de perdas auditivas sensoriais. De acordo com o GATANU, considerou-se como critério de passa a presença de respostas superior a $6 \mathrm{~dB}$ em pelo menos 3 bandas de freqüências (as freqüências testadas foram 1; 1,5; 2; 3 e $4 \mathrm{KHz}$ ) com reprodutibilidade de resposta maior do que $50 \% \%^{11,18}$.

Os resultados da pesquisa foram analisados descritivamente através do cálculo de médias e proporções. Utilizou-se o teste $Z$ de análise de amostras independentes e o teste Odds Ratio para realizar a análise dos resultados obtidos no que diz respeito à perda auditiva. O nível de significância adotado foi de 5\%.

Os dados colhidos foram armazenados e analisados com o auxílio do programa Excel 2002® (Microsoft corporation). Foram calculadas as freqüências específicas das variáveis e as médias, em seguida criadas tabelas binomiais com cruzamentos entre as variáveis estudadas. O teste de regressão logística simples foi utilizado para comparação de proporções, sendo avaliado se existia ou não associação entre duas ou mais variáveis. O grau de significância (valor de p) foi utilizado para quantificar a possibilidade dos números observados apresentarem distribuição ao acaso. Considerou-se como significativo um valor menor do que $5 \%(\mathrm{p}<0,05)$. O Odds ratio foi utilizado para o cálculo da força de associação entre duas variáveis, para quantificar a chance de um fator de risco estar associado ao evento em estudo, com um intervalo de confiança a 95\%. Também foi utilizado o teste $Z$ para comparação entre os resultados obtidos categorizados por idade, gênero, e pela incidência de indicadores de risco para deficiência auditiva. Nesse caso, também foi aceito o nível de significância de 5\% (ou 0.05) para rejeição da hipótese de nulidade.

\section{RESULTADOS}

No período de setembro de 2003 a dezembro de 2006, foram realizadas TAN em 2002 recém-nascidos (RN) em um hospital da rede particular da cidade de Maceió. Destes, 1626 cumpriam os critérios de inclusão, sendo $791(48,6 \%) \mathrm{RN}$ do sexo feminino e $835(51,4 \%)$ do sexo masculino (Figura 1). Dentre os RN do sexo masculino 718 $(44,2 \%)$ passaram na TAN; $13(0,8 \%)$ falharam na orelha esquerda; $8(0,5 \%)$ na orelha direita e $40(2,5 \%)$ falharam nas duas orelhas. No sexo feminino $698(42,9 \%)$ RN passaram; $7(0,4 \%)$ falharam na orelha esquerda; $9(0,6 \%)$ falharam na orelha direita e $16(1,0 \%)$ falharam nas duas orelhas, como pode ser visualizado na Figura 2. Não foi observadauma diferença significativa entre as incidências relacionadas aos gêneros masculino e feminino $(\mathrm{p}=0,3436)$.

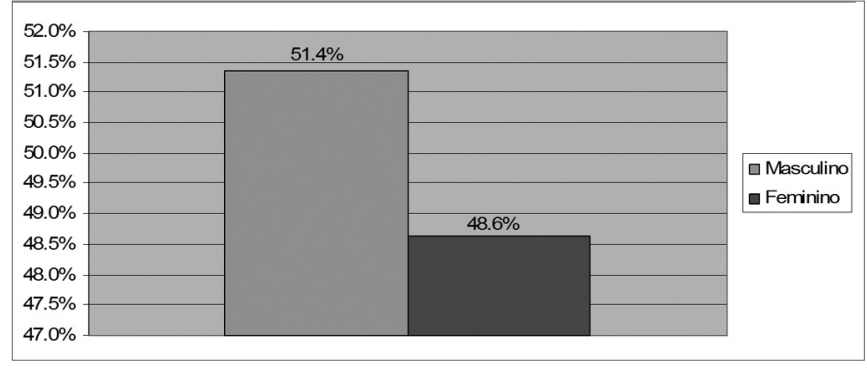

Figura 1. Distribuição percentual da amostra por gênero.

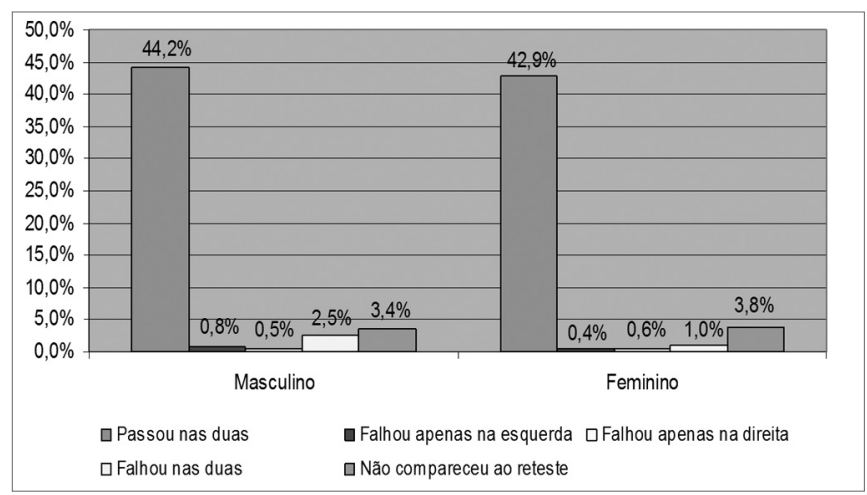

Figura 2. Distribuição percentual dos resultados da TAN por gênero. 
Com relação aos exames realizados por orelha, observou-se que na orelha direita houve 1581 (48,62\%) exames, enquanto que na orelha esquerda foram 1580 (48,59\%), constatando-se que 91 (2,80\%) orelhas não foram avaliadas. A Figura 3 mostra-nos que, na orelha direita, $1488(91,64 \%)$ passaram na TAN e $73(4,49 \%)$ falharam, já na orelha esquerda, $1472(90,59 \%)$ passaram e $76(4,67 \%)$ falharam.Quando aplicado o teste Z, o p foi igual a 0,2961 indicando uma diferença não significativa dos resultados com relação às orelhas.

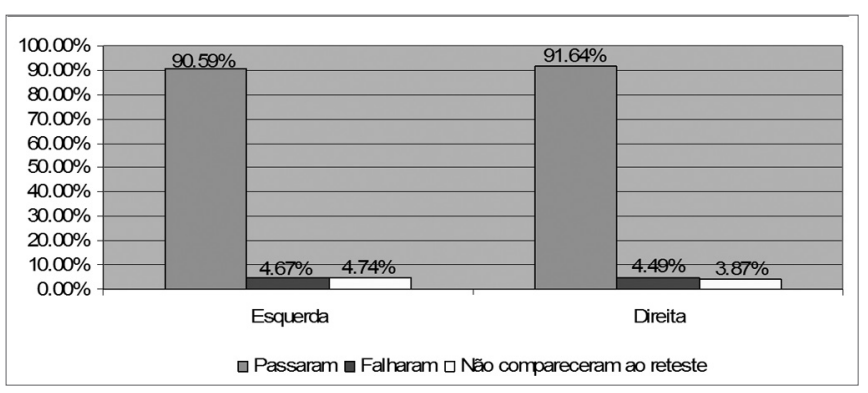

Figura 3. Distribuição percentual dos resultados da TAN por orelha.

A faixa etária mais freqüente em que os RN realizaram TAN, como pode ser visualizado na Figura 4, foi o intervalo de 16 a 30 dias. Este dado apresentou significância, uma vez que, o valor de p foi 0,0427 .

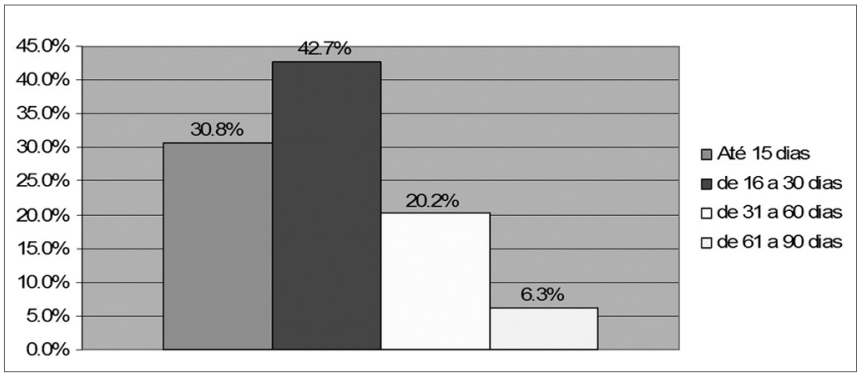

Figura 4. Distribuição percentual da TAN por faixa etária.

Dos $1626 \mathrm{RN}$ atendidos, 1315 (80,9\%) passaram no teste e $311(19,1 \%)$ precisaram ser encaminhados para o reteste. Desses, 194 (11,9\%) compareceram e 117 (7,2\%) não compareceram ao reteste. Com a realização do reteste, observou-se que no total, $1416(87,1 \%)$ RN passaram na triagem auditiva neonatal, enquanto $93(5,7 \%)$ falharam (Figura 5). Dos que falharam, 20 (21,5\%) RN apresentaram ausência de EOAT na orelha esquerda, $17(18,3 \%)$ ausência de EOAT na orelha direita e $56(60,2 \%)$ apresentaram ausência de EOAT em ambas as orelhas. A maior incidência de falhas bilaterais em relação às unilaterais reforça resultados anteriores presentes na literatura ${ }^{19}$. Em relação ao gênero, $61(65,6 \%)$ RN são do sexo masculino e $32(34,4 \%)$ RN são do sexo feminino.

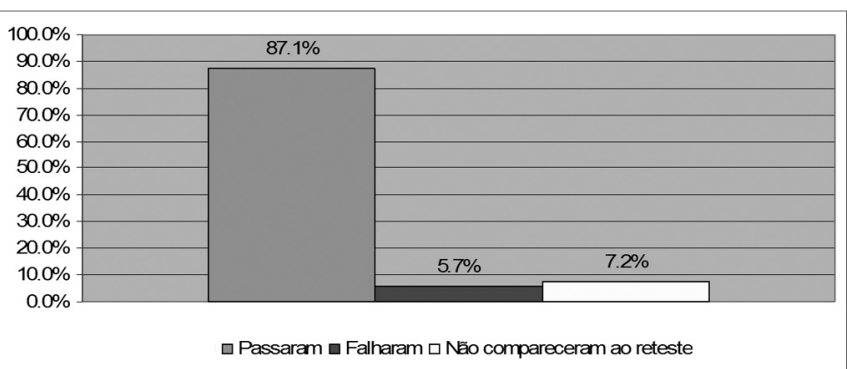

Figura 5. Distribuição percentual da TAN realizada em um hospital do setor privado da cidade de Maceió-AL.

A pesquisa identificou $163(10,0 \%)$ RN com indicadores de risco para deficiência auditiva. Na Figura 6 observa-se que os IRDA mais encontrados durante a triagem foram: a hiperbilirrubinemia com icterícia visível que não necessariamente tenha ido a exosanguíneo-transfusão, seguida de neonatos que ficam mais de 48 horas na UTI neonatal; medicação ototóxica; história familiar de deficiência auditiva congênita; incubadora por mais de 7 dias; ventilação mecânica por período maior que 5 dias; consangüinidade; peso ao nascimento inferior a $1500 \mathrm{~g}$ ou pequeno para idade gestacional; infecção congênita (sífilis, toxoplasmose, rubéola, citomegalovírus, herpes e AIDS); meningite bacteriana e anomalias crânio-faciais. Com uma média de 1,73 indicadores de risco por pessoa.

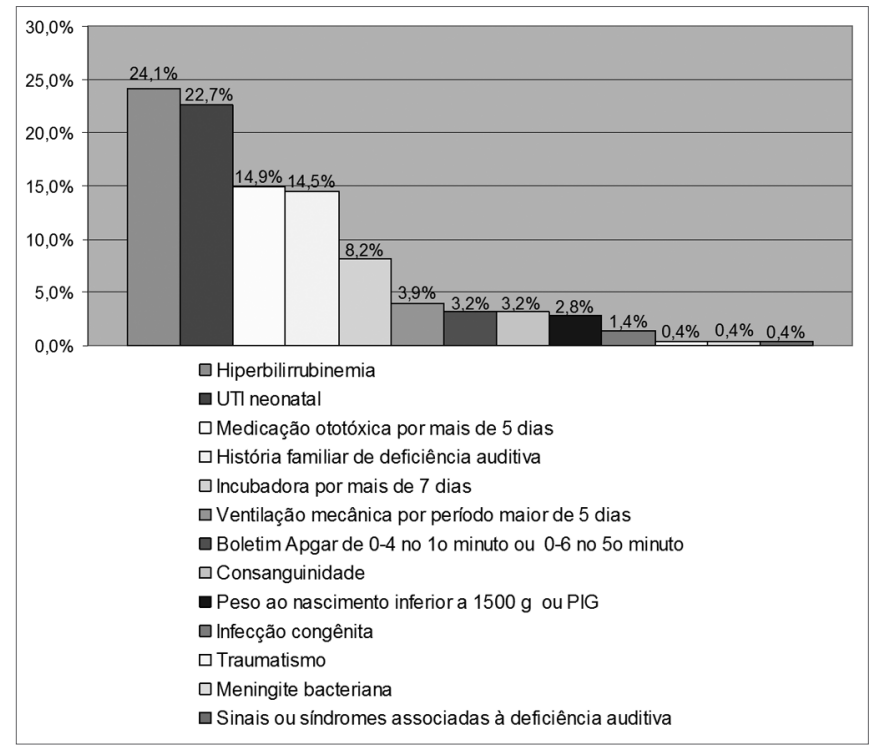

Figura 6. Distribuição percentual dos indicadores de risco para deficiência auditiva.

Dos RN que falharam na TAN, 8,6\% apresentaram algum indicador de risco para deficiência auditiva, enquanto que $91,4 \%$ não apresentaram. Dos que possuem IRDA, $42,9 \%$ utilizaram medicação ototóxica por mais de 5 dias; 21,40\% permaneceram na incubadora por mais de 7 dias; $14,30 \%$ possuem história familiar de deficiência auditiva 
congênita; 7,10\% apresentaram hiperbilirrubinemia com icterícia visível, não necessariamente que tenha ido a exosanguíneo-transfusão; 7,10\% fizeram uso de ventilação mecânica por período maior que 5 dias e 7,10\% permaneceram na UTI neonatal por mais de 48 horas. Perfazendo uma média de 1,75 indicadores de risco por pessoa.

\section{DISCUSSÃO}

No presente estudo, os RN apresentaram EOAT presentes mais no gênero masculino e na orelha direita, porém, ao ser aplicado o teste estatístico $Z$, percebeu-se uma não-significância desses dados. Esse achado difere da literatura pesquisada ${ }^{6,20,21}$, que ressalta uma maior sensibilidade auditiva no gênero feminino e na orelha direita, levando-os a apresentar uma maior prevalência de emissões otoacústicas.

A realização da TAN o quanto antes permite a otimização da intervenção, contribuindo para o desenvolvimento da criança. Na Figura 4, observa-se que a maioria dos RN realizou a TAN no primeiro mês de vida, resultado estatisticamente significante e de acordo com outros autores $^{22,23}$. Esse fato pode refletir que os profissionais da saúde que atuam com bebês demonstram estar atentos às causas, conseqüências e importância da prevenção da deficiência auditiva, encaminhando a criança, precocemente, aos profissionais capacitados, como o fonoaudiólogo e o otorrinolaringologista, para a realização do diagnóstico e/ ou intervenção ${ }^{24}$. A identificação precoce das alterações auditivas possibilita a intervenção ainda no período crítico e ideal de estimulação da linguagem e da audição ${ }^{25}$.

A presente pesquisa apontou que $87,1 \%$ passaram na TAN, $5,7 \%$ falharam e $7,2 \%$ não compareceram ao reteste, apesar da indicação. É importante aqui, entender que o "passar" no teste equivale dizer que, no momento do teste, os resultados foram consistentes com respostas presentes nas EOAT, ou seja, compatível com integridade das células ciliadas externas ${ }^{26,11}$. Ao longo do desenvolvimento infantil, perdas adquiridas decorrentes de: otite secretora, infecções, ototóxicos, causas genéticas ou traumáticas, podem gerar perdas auditivas permanentes ${ }^{26}$.

O CBPAI4 refere que a indicação ao reteste não deve ultrapassar 4\%. A presente pesquisa encontrou um índice de 19,1\%, dado significante segundo teste estatístico $\mathrm{Z}(\mathrm{p}=0.0001)$, inferindo um elevado índice de RN encaminhados para uma nova avaliação. Esse fato pode ser justificado pela possível presença de respiração ruidosa, ato de sucção ou ruído, fatores prejudiciais para captação das EOAT $^{27}$.

Dos RN que apresentaram IRDA observou-se, na Figura 6, que a hiperbilirrubinemia foi o indicador de risco mais freqüente, esse é descrito como uma causa comum que compromete a audição do recém-nascido, podendo lesar a orelha interna e as vias auditivas centrais ${ }^{28}$. O uso de medicação ototóxica vem em segundo lugar, o que diz que, o uso desta é freqüente em berçários. A medicação ototóxica, assim como a consangüinidade, as perdas auditivas genéticas e as doenças infecciosas podem provocar perdas auditivas. O mesmo pode ocorrer com os RN que permanecem em UTI, por apresentarem maior fragilidade, podem ser acometidos por doenças, procedimentos ou medicamentos após a alta do berçário. ${ }^{29}$

De acordo com a literatura, aproximadamente $50 \%$ das crianças diagnosticadas com perda auditiva não apresentam indicadores de risco para a alteração $\mathrm{O}^{27,22}$. Na presente pesquisa, foi observado que $91,4 \%$ dos que falharam não apresentaram indicadores de risco, isso ressalta a importância da triagem auditiva ser universal, ou seja, para todos os RN, possibilitando oportunidades iguais de diagnóstico e intervenção $0^{30,23}$.

\section{CONCLUSÃO}

Este estudo divulgou os resultados estatísticos obtidos em um programa de triagem auditiva neonatal na cidade de Maceió-AL, demonstrando a importância da implantação e manutenção de um programa dessa natureza. Além disso, a sua publicação contribui para estudos multinacionais e/ou regionais.

\section{REFERÊNCIAS BIBLIOGRÁFICAS}

1. Northern JL, Downs MP. Audição em crianças. $3^{\underline{a}}$ ed. São Paulo: Manole; 1989

2. Weber BA, Diefendorf A. Triagem auditiva neonatal. In: Musiek FR, Rintelmann WF. Perspectivas atuais em avaliação auditiva. São Paulo: Manole; 2001. p. 323-38.

3. Pádua FGM, Marone S, Bento RF, Carvallo RMM, Durante AS, Soares JC, et al. Triagem Auditiva Neonatal:um Desafio para sua Implantação. RBORL . 2005;9 (3):189-94.

4. Comitê Brasileiro sobre Perdas Auditivas na Infância (CBPAI). $1^{3}$ Recomendação - período neonatal.J Pediatr Rio de Janeiro:2001:7781

5. Azevedo MF. Programa de Prevenção e Identificação Precoce dos Distúrbios da Audição. Processamento Auditivo (Schochat, E.). Rio de Janeiro: Lovise; 1996. p. 75-100.

6. Durante AS, Carvallo RMM, Costa MTz, Cianciarullo MA, Voegels RL. Triagem Auditiva Neonatal - justificativa, possível e necessária. RBORL . 2003;69 (2):11-8.

7. Kemp DT, Ryan S. Otoacoustic emission tests in neonatal screening programmes. Acta Otolaryngol . 1991;(Suppl 482):73-84.

8. Kemp DT. Otoacoustic emissions: basic faces and applications. Audiol Pract . 1989;6:1-8.

9. Write KR, Vohr BR, Behrens R. universal new born hearing screening using transient evoked otoacoustic emissions: results by the Rhode Island Hearing Assessment Project. Semin Hear . 1993;14:18-29.

10. Bonfils P. Screening for auditory dysfunction in infants by evoked otoacoustic emissions. Arch Otolaryngol Head Neck Surg . 1988;114:88790.

11. GATANu - Grupo de apoio a triagem auditiva neonatal universal. Disponível em http://www.gatanu.org. Acessado em 10 de julho de 2007.

12. Lopes-Filho O. Deficiência auditiva. In:Lopes-Filho O. Tratado de Fonoaudiologia. São Paulo: Roca; 1997. p. 3-24.

13. Bonfils P. Spontaneous and evoked otoacoustic emissions in preterm neonates. Laryngoscope . 1992;102:182-6. 
14. Hatzopoulos S, Prosser S, Mazzoli M, Rosignoli M, Martini A. Clinical applicability of transient evoked otoacoustic emissions: identification and classification of hearing loss. Audiol Neurootol . 1998;3:402418.

15. Decreton SJRC. Evoked otoacoustic emissions in infant hearing screening. International. Int J Pediatr Otorhinolaryngol . 1991:21:23547.

16. Joint Committee on Infant Hearing (JCIH), 2000. Position statement Disponível em:<http://www.audiology.org/professional/positions/ jcih-early.php>. Acessado em 18 de julho de 2007.

17. Joint Committee on Infant Hearing (JCIH), 1994. Position statement Disponível em:<http://www.audiology.org/professional/positions/ jcih-early.php>. Acessado em 15 de julho de 2007.

18. Finitzo T, Albright K, O'Neal J. The newborn with hearing loss: detection in the nursery. Pediatr . 1998;102:1452-1460.

19. Soares E, Guerrem SMA;Azevedo MF. Estudo Comparativo das Triagens Auditivas por Emissões Otoacústicas Evocadas Transientes, Observação Comportamental e Medidas de Emitância Acústica em Crianças com e sem Risco para Deficiência Auditiva. RBORL . 1998;64 (3):221-7

20. Kannan PM, Lipscomb DM. Bilateral hearing asymmetry in a large population. J Acoust Soc Am . 1974;55 (5):1092-4.

21. Durante AS. Estudo da amplitude das emissões otoacústicas evocadas transitórias com ruído contralateral em lactentes. [dissertação]. São Paulo (SP): universidade de São Paulo; 2000.
22. Baroch KA. universal newborn hearing screening: fine-tuning process Curr Opin Otolaryngol Head Neck Surg . 2003; 11:424-7.

23. Joint Committee on Infant Hearing. Year 2000 position statement. Principles and guidelines for early detection and intervention programs. Am J Audiol . 2000; 9(1):9-29.

24. Dell' Aringa AHB, Oliveira VV, Mello JM, Oliveira JRM. Deficiência Auditiva: suspeita e identificação. Pediatria Moderna . 2005;41(5):251-4.

25. Northern JL, Downs MP. Behavioral hearing testing of children. In Hearing in children. 4. ed. Baltimore: 1991.

26. Widen JE, Bull W, Folsom RC. Newborn Hearing Screening: What it means for providers of early intervention services. LWW/IYC 2003;16(3):249-57.

27. Ribeiro FM. Programa de Triagem Auditiva Neonatal. In: Hernandez AM, Marchesan. I Atuação Fonoaudiológica no Ambiente Hospitalar. Rio de Janeiro: Revinter; 2001. p.152-5.

28. Almeida FS, Pialarissi PR, Monte Alegre AC, Silva JV. Emissões acústicas e potenciais auditivos evocados do tronco cerebral: estudo em recém-nascidos hiperbilirrubinêmicos. RBORL . 2002;68:851-8.

29. Dell'aringa AR. Contribuição ao estudo das disacusias hereditárias, progressivas e de causas desconhecidas. [Tese Doutorado]. Faculdade de Medicina da universidade de São Paulo; 1999.

30. Yoshinaga-Itano C, Sedey A. Language, speech, and social-emotional development of children who are deaf or hard of hearing: the early years. The Volta Review 2000;100(5):298. 